\title{
Reforma, autonomía universitaria e interés nacional: análisis de la cátedra de Economía Política en la Universidad Nacional de Colombia, Bogotá 1933-1938 ${ }^{1}$
}

\author{
José Molina Bravo ${ }^{2}$ \\ Universidad de Arte y Ciencias Sociales (Chile) \\ Universidad Autónoma de Colombia (Colombia) \\ Grupo de investigación HISULA \\ jamolinab@gmail.com
}

Recepción: 14/06/2013

Evaluación: 28/11/2013

Aceptación: 13/12/2013

Artículo de Reflexión

DOI: http:/ / dx.doi.org/ 10.9757/Rhela.22/05

\section{RESUMEN}

Este trabajo plantea una aproximación a la Reforma Universitaria desde una perspectiva sociopolítica al describir la importancia de las cátedras y el lugar que en el proceso de cambio se le asigna a los procesos de formación académica, profesional e intelectual. Aborda el caso de la Universidad Nacional de Colombia, en la cátedra de Economía Política de la Facultad de Derecho y Ciencias Políticas (1933-1938). Utiliza fuentes de archivo para caracterizar el ambiente de Reforma, las discusiones generadas en la Facultad mencionada y la forma en la que se organizó la enseñanza de la economía política. En su análisis, combina planteamientos sobre el campo intelectual-universitario, la perspectiva social del fenómeno educativo y el análisis crítico del discurso.

Palabras clave: Revista Historia de la Educación Latinoamericana, reforma universitaria, autonomía, cátedra, economía política, Universidad Nacional de Colombia.

1 Este manuscrito se articula al proyecto Reformas y movimientos universitarios en Colombia SGI 1295, desarrollado por el grupo Ilustración en América Colonial, ILAC y financiado por la Dirección de Investigaciones de la Universidad Pedagógica y Tecnológica de Colombia.

2 Doctorando en el Doctorado en Procesos Sociales y Políticos de América Latina de la Universidad de Arte y Ciencias Sociales, Magíster en Sociología, Docente e Investigador de la Facultad de Ciencias Humanas, Programa Historia, Universidad Autónoma de Colombia e integrante del grupo de investigación HISULA de la Universidad Pedagógica y Tecnológica de Colombia. 
“Reform, university autonomy and national interest: analysis of the chair of political economy at the National University of Colombia, Bogota 1933 - 1938"

\section{ABSTRACT}

This paper presents an approach to the University Reform from a sociopolitical perspective. It describes the importance of the chairs and place that in the process of change is assigned to the processes of academic professional and intellectual development. It takes into account the case of the Ntional University of Colombia, in the chair of Political Economy, of the Faculty of Law and Political Science (1933 - 1938). The sources are taken from a file to characterize the atmosphere of reform, the discussions generated in the above-mentioned option and the way it has organized the teaching of political economy. In its analysis there are mixed approaches on the intellectual field - university, the social perspective of the phenomenon of education and critical discourse analysis.

Key words: Journal History of Latin American education, university reform, autonomy, chair, economic policy, National University of Colombia.

\author{
“Reforma, autonomia universitária e interesse \\ nacional: Análise da cátedra de economia \\ política na Universidade Nacional da Colômbia, \\ Bogotá 1933-1938"
}

\section{RESUMO}

Este trabalho promove uma aproximação à Reforma Universitária a partir de uma perspectiva sociopolítica, descrevendo a importância das cátedras e o lugar que, no processo de mudança, se lhe determina os processos de formação acadêmica, profissional e intelectual. Aborda o caso da Universidade Nacional da Colômbia, na cátedra de Economia Política, da Faculdade de Direito e Ciências Políticas (1933-1938). Utiliza fontes de arquivo para caracterizar o ambiente de Reforma, as discussões geradas na Faculdade mencionada e a forma em que se organizou o ensino da economia política. Em sua análise, combina explicações sobre o campo intelectualuniversitário, a perspectiva social do fenômeno educativo e a análise criticado discurso.

Palavras-chave: Revista História da Educação Latino-americana, reforma universitária, autonomia, cátedra, economia política, Universidade Nacional da Colômbia.

\section{INTRODUCCIÓN}

Este trabajo describe una característica de la transformación de la reforma universitaria de la Universidad Nacional de Colombia, entre 1933 y 1938, en un problema de gobierno, y de la conversión de los intelectuales, científicos y profesionales en actores políticos. Abordará el contexto institucionaluniversitario en el que sus procesos de formación académica asumieron el mismo carácter político y gubernamental, más aún cuando las fuerzas liberales triunfantes señalaron que la Universidad tenía una deuda moral con el pueblo, que debía refundarse para servir a la nación. Al respecto, se consultaron Actas del Consejo Directivo de la Universidad Nacional correspondientes al período; Programas del curso de Economía Política y artículos referidos a la Reforma Universitaria, publicados en la Revista Jurídica de la Sociedad Jurídica de la Facultad de Derecho y Ciencias Políticas de la Universidad Nacional de Colombia en 1935. 
La reforma de la universidad es un proceso de cambio político que incide directamente en la notoriedad pública de los "intelectuales" y su acción en el campo del poder. La reforma del espacio intelectual, en las primeras décadas del siglo XX, convirtió al sistema educativo en un escenario de conflicto entre los valores meritocráticos y las "leyes de reproducción de las élites", tensión que se expresó en los debates sobre las profesiones y la función legitimadora o crítica de los intelectuales en la construcción de sociedades nacionales y su democratización. ${ }^{3}$

Durante el gobierno de la Revolución en Marcha (1934-1938), el carácter político de la enseñanza profesional universitaria, en el marco de reforma de la Universidad Nacional de Colombia, se refleja claramente en la discusión sobre su función social, su carácter nacional y su autonomía. No obstante, es menos visible cuando la 'ejecución' de la reforma se enfoca en la vida interna de la institución. En ese nivel, las facultades y cátedras reflejaron las tensiones, cambios y estructuras nuevas en las que se expresó tal reforma.

Específicamente, la cátedra de economía política de la Universidad Nacional se transformó en un escenario singular donde el Estado y la Universidad instauraron orientaciones sobre la enseñanza de la economía y su importancia para el estudio, la comprensión y la solución de los problemas nacionales. Un ejemplo es la participación como profesor del propio presidente electo en 1934, Alfonso López Pumarejo. Su intervención ubicó a la enseñanza de la economía política en la frontera de dos períodos de la reforma. Entre 19321934 la reforma universitaria se expresó como iniciativa intelectual y de debate político; luego, entre 1935-1938 la refundación de la Universidad tomó un carácter legislativo y gubernamental mediante la discusión y aprobación de la Ley 68 de 1935 y la construcción de la Ciudad Universitaria. (1937)

\section{La cátedra de Economía Política en el proceso de Reforma Política y Universitaria}

El 4 de julio de 1933, el profesor Horst Rollitz, titular de la cátedra de economía política, dictó una conferencia en el salón de actos de la Facultad Nacional de Derecho y Ciencias Políticas de la Universidad Nacional de Colombia, la cual fue titulada Problemas económicos colombianos. ${ }^{4}$ Este nombre lo incluyó en

3 Christophe Charle, Naissance des "intellectuels" (París: Les Éditions de Minuit, 1990).

4 Horst Rollitz, "Problemas económicos colombianos", Revista Javeriana 3 (1933): 121-127. 
una doble tradición: por una parte, la de los intelectuales colombianos que, como Alejandro López, pensaron la realidad nacional y las tareas de la clase política dirigente en el desarrollo del país, y, por otra, se insertó también en la tradición del científico social que desde una noción de economía nacional señalaba las tareas del Estado en el estímulo de la actividad productiva y el aprovechamiento de las capacidades de la población. ${ }^{5}$

En esta conferencia, el Dr. Rollitz ${ }^{6}$ se refirió a los problemas económicos que afectaban a los países que denominó como "semi-coloniales", condicionados por la falta de fundamentos para la vida económica. En su diagnóstico de la realidad colombiana resaltó la "limitada densidad poblacional" y su desigual distribución en el territorio, la necesidad de una reforma agraria mediante la intervención del Estado y de incentivar la inmigración "de ciertos elementos extranjeros, adecuados al carácter del pueblo colombiano". ${ }^{7}$ Como soluciones, planteó la importancia de superar técnicamente los obstáculos geográficos y de "fomentar un racional sistema de vías de comunicación, base fundamental de toda economía moderna". También propuso fomentar el turismo, la producción agrícola, la exportación del café y la minería.

Para el profesor Rollitz los factores dinámicos de la modernización de la economía colombiana eran tres: primero, la formación de capital para estimular el desarrollo económico y social; segundo, la renuncia a la aplicación de ideas socialistas al ser estas "teorías irrealizables o perniciosas, mediante cuya aplicación, en el actual desarrollo de la raza humana, no se obtendría otro resultado que echar por tierra la Economía, esterilizando así las penosas labores de varias generaciones" $y$, en tercer lugar, garantizar mediante conocimientos e iniciativas económicas la armonía y alianza de las fuerzas productivas. ${ }^{8}$

Meses antes de la conferencia reseñada, Rollitz, un “técnico alemán contratado por el gobierno nacional para explicar la cátedra de Ciencias Económicas en la Facultad de Derecho", había dictado voluntariamente otra conferencia sobre la crisis económica mundial, en la que demostró su "autoridad científica",

\footnotetext{
5 Al respecto: Max Weber, "El estado nacional y la política económica”, en Obras Selectas, (Buenos Aires: Distal, 2010).

6 Entre las reseñas de libros realizadas por Félix Restrepo, se presentó la tesis doctoral del profesor Rollitz, señalando lo siguiente: "El Dr. Horst Rollitz ha hecho largos estudios de Economía en Berlín bajo la dirección del profesor Hermann Schumacher, y está actualmente contratado por nuestro gobierno para impulsar los estudios económicos en la República". Félix Restrepo, "Revista de libros: Recent social trends, Akerman, Becerra, Bruce, Edie, Einzig, Rollitz, Uribe", Revista Javeriana (1933): 90.

7 Rollitz, "Problemas económicos colombianos", 122.

8 Rollitz, "Problemas económicos colombianos", 124-125. En el punto de la armonía entre clases y/o fuerzas productivas, la exposición del Dr. Rollitz también emparenta con la tradición de pensamiento sociológico y económico colombiano expresada en los planteamientos de Miguel Samper, Salvador Camacho Roldán y Alejandro López, entre otros.
} 
aportó a la comprensión universitaria de los problemas nacionales y, de paso, reforzó que las universidades

son organismos de vanguardia en el progreso de las ideas, con aplicación permanente al bien público, no solo por la misión propia de difundir y aumentar la cultura, cuanto por el estudio $y$ análisis que en ellos se hace de las cuestiones de actualidad que revisten importancia para la vida del respectivo país [... ..$^{9}$

La enseñanza de la economía se tornó en un espacio en el cual la nación y la sociedad fueron objeto de diagnósticos, análisis y valoraciones. La realidad nacional fue considerada como criterio de valoración de la enseñanza, orientó el debate sobre el desarrollo disciplinario y sobre su carácter científico. Los problemas nacionales definieron la pertinencia de los contenidos formativos en economía, presionando por la 'adecuada' composición del lenguaje y el discurso disciplinar. Influyeron decisivamente en la institucionalización de la formación universitaria de economistas -contra los banqueros y hombres de negocios- y también en la selección de las escuelas de pensamiento económico que definieron los procesos de enseñanza.

En este período se inició la transición hacia la extensión y consolidación de programas universitarios de economía, sin embargo, en la Universidad Nacional esta disciplina siguió siendo parte de la carrera de Derecho. ${ }^{10}$ Ubicada en el pénsum en el primer año de formación en Derecho (1932 a 1936), y a cargo del Profesor Horst Rollitz, la enseñanza de la Economía Política abordó el pensamiento económico mediante la revisión de "escuelas", la moralidad de la vida económica y su estatus científico. ${ }^{11}$ Se consideró como parte del programa de estudios la relación entre Economía Política y Derecho, identificando como componentes de su 'objeto de estudio' a la actividad económica, su racionalidad y tipología para diferenciarlos de las nociones de orden jurídico, costumbre y moral, lo cual se hizo extensivo hasta el vínculo entre economía y religión. Esta revisión continuaba con la identificación de los motivos económicos

9 Fernando Rhenals, “Las ideas del profesor Rollitz sobre la crisis económica mundial”, Revista Jurídica 229-230 (1933):134.

10 Álvaro Montenegro, Los primeros programas de economía en Colombia, (Bogotá: Universidad Javeriana, 2008). Consultado el 18 de septiembre de 2012 http:/www.javeriana.edu.co/fcea/area_economia/inv/documents/ LosprimerosprogramasdeeconomiaenColombia.pdf.

11 Jose Molina Bravo, "Programa del curso de Economía Política dictada en la Facultad Nacional de Derecho y Ciencias Políticas por el Profesor Horst Rollitz, durante el año 1933”, (AC UNAL), Nómina de Programas, proyectos, quejas, reglamentos 1927-1935, Caja 37. 
individuales y su diferencia específica con las intervenciones estatales. Mientras la racionalidad de la acción económica era individual, la acción del estado en el campo económico fue caracterizada como asistencia, caridad e intervención social. Esto se complementó con la enseñanza de la noción técnica de mercado.

La definición del objeto de estudio de la economía implicaba la propia delimitación de la noción economía nacional. Esto evidenció una doble inscripción del programa del curso, por una parte, la influencia de la Escuela Romántica y la Escuela Histórica alemana -que contextualiza la revisión de los planteamientos de List y Schmoller, entre otros- en el aprendizaje de las doctrinas económicas; por otra, la inscripción de la racionalidad del fenómeno económico en escalas doméstica, nacional, mundial. Asimismo, la relación de la economía con la política fue abordada a partir de las nociones de "nacionalismo económico moderno" -incluyendo contenidos ligados a los factores raciales y morales con base en la categoría de pueblolo cual identificaba tanto la función del Estado en el desarrollo del país como las formas de inserción en la economía mundial.

El programa del curso de Economía Política que dictó el profesor Rollitz junto con definir la tipología de la acción económica y su consecuente racionalidad vinculóla enseñanzay la acción en el campo económico a un problema histórico-político, lo cual demuestra la coherencia de la operación que delimita un objeto epistemológico en la enseñanza de la disciplina. Esto fue complementado con la revisión de la trayectoria de la ciencia económica. Rollitz presenta la historicidad del pensar económico y de sus principios, coherentes con la racionalidad de la acción económica, como el contenido que evoluciona. Lo historiza mediante una periodización clásica de antigüedad, edad media, edad moderna. Luego, delimita escuelas de pensamiento económico, al utilizar contenidos que le permiten describir -transponer- un objeto de aprendizaje: por un lado, el pensamiento mercantilista y las condiciones históricas de aparición de su pensar el problema de la nación y la intervención del Estado. Procede de igual manera con la doctrina de los fisiócratas, su pensar librecambista, sus conceptos de Estado y de orden natural, así como el problema histórico -económico de la oposición entre agricultura y comercioindustria que equivale a su descripción de la oposición conceptual entre campo y ciudad. 
El gesto científico-pedagógico del Dr. Rollitz se consolida cuando la revisión de la historia de la ciencia económica se torna contemporánea. Este carácter de su abordaje radica en la propuesta de un segundo orden de observación de la propia evolución disciplinaria desde el punto de vista doctrinario. Si el pensar económico tiene condiciones históricas de aparición y reflexión, entonces sus objetos epistemológicos y de aprendizaje cercanos al presente fueron influenciados por el problema del vínculo entre racionalidad económica (individual) y el desarrollo de la nación, determinantes del carácter moral del problema y de su propia escala de análisis.

Entre el capítulo cuarto y sexto del libro tercero de su programa de contenidos, Rollitz sitúa su estrategia de análisis y pedagógica en la realización de balances de autores, desarrollos conceptuales, progresos y deficiencias de la "La Escuela Clásica de la Ciencia de la Economía Política" y en particular de Adam Smith. Luego, introduce los planteamientos del pensamiento romántico alemán (List), la utilidad marginal y el socialismo. Polemiza, evalúa, distingue, clasifica, categoriza. Con estas operaciones, Rollitz propone un abordaje de las teorías y doctrinas económicas cuyo método se aproxima a la investigación científica.

Este programa de contenidos se desarrolló en el contexto de crítica a los métodos de educación que emergió en las Facultades en el proceso de reforma universitaria. Por ello, su eficacia y pertinencia pedagógica sería evaluada en un medio universitario, presionado por adecuarse a las condiciones históricas e institucionales que le permitieran cumplir con la función social de la universidad. Las cátedras, por lo tanto, se transformaron en espacios políticos no solo porque presidentes, funcionarios estatales, líderes políticos y empresariales estuvieran al mando de los procesos de formación académica, su política particular se trasladó desde los individuos a las formas de enseñanza y transformó a las comunidades académicas en actores que disputaron la conducción de las nuevas orientaciones que exigía la reforma, la nueva universidad y la nación. 


\section{Revolución en marcha y reforma de la Universidad Nacional}

Lasreformas alaUniversidad Nacional duranteel gobierno de LópezPumarejo se dirigieron hacia tres ámbitos: su refundación mediante la reivindicación de la integralidad de las funciones de la Universidad; su centralización con la consiguiente relativización de su autonomía y el cambio en el esquema de profesiones que reorganizó la enseñanza, la función de la ciencia y la vida académica de sus facultades y escuelas. La formación de profesionales en el sentido y espíritu integrador de las funciones universitarias.

Para el gobierno liberal, la autonomía de la Universidad no era un problema. Tenía una clara posición al respecto. Esta autonomía debía ser relativa. Tal como lo enunció López Pumarejo en su intervención en la cátedra de Economía Política ${ }^{12}$ y luego Carlos García Prada en la exposición de motivos del proyecto de Ley Orgánica de la Universidad Nacional, la autonomía universitaria reforzaba la libertad de cátedra, siendo la enseñanza un ámbito en el cual el Estado no intervendría. ${ }^{13}$ Sin embargo, en el ámbito del gobierno de la Universidad, su autonomía absoluta era "imposible":

\section{[...] es cierto que la Universidad necesita de una autonomía rela- tiva que le permita vivir y crecer dentro desu propio ambiente y dentro de las circunstancias especialísimas de orden fiscal en que se encuentra y habrá de encontrarse en el curso de muchísimos años.}

La razón fundamental de esta decisión estatal fue la necesidad del "equilibrio orgánico" de la Universidad que, según esta visión, dependía de la conducción, "acción creadora y fiscalizadora del Gobierno". ${ }^{14}$

El propósito ejecutivo de fondo fue la centralización de la Universidad, dictaminada por la Ley 68 de 1935, específicamente en su artículo $1^{\circ}$ que la definió como "una persona jurídica dentro de las normas de la Constitución y de la presente Ley". Este principio fue considerado como una garantía insoslayable para que la propuesta de reforma académica realmente 'innovadora', a saber,

\footnotetext{
12 El Tiempo, "López Pumarejo y su cátedra libre de Economía Política”, en Alfonso López Pumarejo y la Universidad Nacional de Colombia editado por la Universidad Nacional de Colombia, 42-43.

13 Al finalizar la exposición de motivos del Proyecto de Ley “orgánica de la Universidad Nacional”, Carlos García Prada señaló que "En materia de enseñanza, la educación humana es de importancia capital, y ni la inteligencia, ni la voluntad, ni la imaginación creadora, ni la preparación de maestros y discípulos son cosas sobre las cuales puede legislar el Congreso de la República”. Carlos García Prada, "Exposición de motivos", en Alfonso López Pumarejo editado por la Universidad Nacional de Colombia, 91.

14 García Prada, "Exposición de motivos”, 71-72.
} 
la "división departamental de las Facultades y Escuelas", pudiera aplicarse sin poner en riesgo la unidad orgánica de la institución:

Es urgente que la Universidad se divida, ante todo, en tantas unidades subordinadas y coordinadas como sean necesarias, y que ellas sean la suma activísima de funciones vitales y culturales a cada una de las cuales corresponda una unidad administrativa estructural en símisma, dotada de una adecuada independencia. ${ }^{15}$

En términos académicos, la división propuesta incluyó la formación de Facultades, Escuelas y Servicios, divididos a la vez en departamentos correspondientes a las diferentes áreas de conocimientos y disciplinas, concentrando también sus respectivas funciones de docencia, investigación, publicación y servicios:

El departamento universitario de enseñanza y de investigación específicas, que puede estar a cargo de uno o más profesores, según las circunstancias, necesita de autonomía administrativa y pedagógica (y también de un profesorado idóneo, responsable y entusiasta), para que su funcionamiento sea flexible y progresista. ${ }^{16}$

La reforma de la Universidad Nacional en su fase legal (1935 en adelante) modificó el esquema de profesiones, resultado de su "autonomía universitaria relativa"; desplazó el debate desde el campo político hacia el de la enseñanza, que fue la principal preocupación del gobierno liberal. Con ello, a partir de la Reforma de 1935 y en los sucesivos gobiernos, el

tradicional esquema de profesiones compuesto de medicina, derecho e ingeniería fue ampliado a nuevas actividades técnicas y científicas. Se crearon entonces nuevas facultades: química, arquitectura, veterinaria, agronomía, economía, administración, filosofía, etc., a las cuales se agregaron algunos institutos de investigación como el de ciencias naturales. ${ }^{17}$

Esta ampliación de la estructura académica, científica y profesional creó un medio universitario con capacidad estructural para abordar progresivamente

\footnotetext{
15 Ibid., 77.

16 Ibid., 80.

17 Jaime Jaramillo Uribe, “La educación durante los gobiernos liberales. 1930-1946”, en Nueva Historia de Colombia, dirigida por Álvaro Tirado Mejía (Bogotá: Planeta, 1989), 107.
} 
la realidad nacional. De esto dio cuenta, por ejemplo, el profesor Dr. Pérez Arbeláez que abogó ante el Consejo Directivo de la Universidad Nacional para la creación del Instituto Botánico:

\begin{abstract}
Dice que el proyectado Instituto Botánico no requiere para su instalación muchos gastos, ya que lo más urgente sería montar un laboratorio químico para estudiar las diversas plantas y acometer el análisis de los suelos. El herbario nacional debe pertenecer al instituto, cuya necesidad más urgente por ahora es un local donde funcionar independientemente. Dentro de los planos que están elaborándose para la ciudad Universitaria [sic], hay varios parques que podrían cederse y destinarse a Jardín Botánico [...] Termina diciendo que la Universidad Nacional, al acoger la iniciativa de fundación del Instituto Botánico, daría un hermoso ejemplo de vinculación con la riqueza nacional [...] El señor Rector de la Universidad manifestó que estaba listo a dar toda clase de facilidades al Profesor Pérez Arbeláez en sus trabajos y ofreció cederle un local en el edificio que actualmente ocupa la Escuela de Ingeniería e incluir la partida necesaria en el proyecto de presupuesto de la Universidad que presentará próximamente al Consejo Directivo. ${ }^{18}$
\end{abstract}

La reforma logró hegemonizar la actividad universitaria y convertirse en una lógica de desarrollo que al salir del ámbito de la discusión partidaria y gubernamental, se insertó en la vida de las Facultades, Institutos y Escuelas. Los órganos superiores definidos por la Ley 68 de 1935, el Consejo Directivo y el Consejo Académico, tomaron las riendas del funcionamiento universitario, asumiendo la responsabilidad de ejecutar el cambio institucional y académico que propiciara el aumento de la cientificidad en la formación profesional y, a la vez, integrara en los actos de enseñanza todas las funciones de la Universidad para dar cumplimiento a su servicio a la Nación. Este imperativo se materializó en la construcción de la Ciudad Blanca, el campus universitario que debía reunir a las distintas Facultades y Escuelas y hacer realidad el encuentro de los estudiantes y las disciplinas científicas. ${ }^{19}$

18 Acta n 12 Sesión del 12 de mayo de 1936 (Bogotá, 1936), Archivo Central Universidad Nacional de Colombia (AC UNAL) Actas Consejo Directivo Universidad Nacional de Colombia, 38.

19 Clara Sánchez Botero, "Ciencia y educación superior en la República Liberal", en República Liberal: sociedad y cultura, Editor Rubén Sierra Mejía (Bogotá: Universidad Nacional de Colombia, 2009), 532 - 534. 


\section{La Reforma Universitaria y la Facultad Nacional de Derecho}

Hacia 1935, la Facultad Nacional de Derecho y Ciencias Políticas había dejado su 'vida de claustro' para abrirse a los problemas nacionales; en el ámbito académico y político el ambiente reformista impulsó a sus estamentos a debatir y evaluar el avance y los sentidos del cambio. En junio de ese año Eliseo Arango, rector de la Facultad, envió su informe al Ministro de Educación en el que exponía "algunas verdades que son el patrimonio común de las gentes". En septiembre, Guillermo Tello Rengifo, uno de los directores de la Revista Jurídica y socio numerario de la Sociedad Jurídica de la Universidad Nacional, planteó el problema de la “democratización de la enseñanza universitaria"; en octubre, fue presentado el Informe solicitado por la Sociedad Jurídica a la "Comisión encargada de estudiar el proyecto de 'Ley Orgánica de la Universidad Nacional de Colombia'”. En estos tres actos se aprecia el vínculo entre los contenidos ideológicos de la reforma universitaria y los aspectos que se han destacado de la enseñanza de la economía política.

En primer lugar, el diagnóstico de Eliseo Arango, exministro de Educación y Rector de la Facultad Nacional de Derecho y Ciencias Políticas en 1935, planteó sus serias dificultades materiales para ser

un centro de investigación cientifica y de preparación de profesionales idóneos, para el país y su tiempo [...] La Facultad se encuentra en condiciones precarias para realizar estos fines, y existen circunstancias adversas que sólo pueden ser superadas con una labor sin pausa y sin precipitación..$^{20}$

Junto con el déficit de infraestructura y de investigación verdaderamente científica, el Rector identificó problemas para que profesores y estudiantes pudieran cumplir con los objetivos de una formación "profesional con perspectivas"; a tales obstáculos los llamó un "círculo vicioso" que por el lado de los profesores se reproducía por su falta de estímulo económico para comprometerse con el cumplimiento de la misión de la Universidad. Los estudiantes, por su parte, señaló el Rector.

20 Eliseo Arango, "Informe del Rector de la Facultad al Ministro de Educación Nacional”, Revista Jurídica nº 236 (1935): 7. 
"tienen cualidades y defectos considerables. Resalta ante todo la inteligencia clara y ágil, propia de la mentalidad mediana de nuestra raza, pero carecen de disciplina, de precisión, de método. Viven, por ello, sin los fundamentos de un 'buen jurista', en una "simulación de la cultura". ${ }^{21}$

A partir de esto, el rector Arango relevó la necesidad de que la reforma universitaria se desarrollara sobre bases técnicas, criticando el planteamiento de la autonomía relativa realizado por el gobierno:

La actividad del Estado en estos particulares consiste en dotar a nuestros institutos de los elementos que exige el desarrollo de la cultura. La "nueva orientación ideológica" de que tanto se habla, surge de los laboratorios, de las salas de estudio, de la pasión ardiente por encontrar la verdad. Es infantil la pretensión de que el poder público pueda fijar esos objetivos. ${ }^{22}$

Además de reivindicar la autonomía universitaria y la no imposición de criterios gubernamentales en la definición de su estatus jurídico, su moral, su filosofía y su economía, Eliseo Arango refrendó la necesidad de adaptar el "pénsum a las nuevas necesidades que se presentan a la vida del abogado (sic)", para lo cual era necesario conocer el ambiente profesional, definir especializaciones y priorizar la formación a los estudiantes para la vida.

Para evidenciar su diagnóstico, el Rector de la Facultad enunció un conjunto de medidas tomadas: creación de cursos libres, organización de salas de trabajos prácticos para las materias de Derecho Civil, Comercial, Penal, Cuestiones Económicas, Ciencias Políticas y Sociales con el objeto de estimular la investigación científica entre los estudiantes, el mejoramiento de la biblioteca, entre otras. Estas actividades fueron reconocidas en el momento por profesores quienes valoraron los cambios en los estudios de Derecho Civil y la "revolución en relación con los estudios de Derecho Social contemporáneo", con la incorporación de estudios sobre "legislación industrial y obrera". ${ }^{23}$

21 Arango, Informe del Rector, 7- 8.

22 Arango, Informe del Rector, 9

23 Bayé, "La actual universidad. Progresos de "Santa Clara". - El movimiento universitario. Entrevista con el doctor Rodrigo Jiménez Mejía”, Revista Jurídica nº 237 - 238 (1935): 278. 
Este diagnóstico y las medidas de reforma del pénsum apuntaron al problema de la profesionalización en la formación de abogados, conflicto corroborado en el mismo año por la protesta de estudiantes ante el aumento de los derechos de matrícula, lo que de consuno se interpretó como un problema social y político que debía ser asumido por la reforma universitaria. Según Guillermo Tello Rengifo, este problema correspondía a

La protección a los profesionales existentes. Ello es asi porque al estudiante colombiano las mayores trabas que se le interponen son en la mayoría de los casos insubsanables, dada la escasez de recursos de la clase media económica. De un lado los derechos de matrícula excepcionalmente elevados; de otro el altísimo precio de los libros de estudio $y$ de consulta y de instrumentos de trabajo. Si a ello se añade el precio exorbitante de la vida en Colombia, tenemos que la enseñanza universitaria sólo puede seguirse con regularidad por los jóvenes pertenecientes a familias ricas. ${ }^{24}$

Por su parte, la Comisión que estudió el proyecto de Ley Orgánica expresó su rotunda satisfacción con la presentación, considerando que garantizaba un próspero futuro para la Universidad Nacional al promover su centralización y la articulación de las Facultades, su departamentalización y la trascendencia de los servicios e institutos para el cumplimiento de funciones olvidadas por la universidad. Celebró la proposición estatal de conceder autonomía relativa a la universidad, ya que no era posible dar autonomía absoluta a "una universidad que, puede decirse, aún no existe". ${ }^{25}$

No obstante, esta visión positiva de la iniciativa estatal se fundó en una premisa común a las anteriores visiones y diagnósticos: el proyecto de Ley Orgánica de la Universidad Nacional enfrentaba decididamente los problemas de la enseñanza profesional. Para esta comisión, el diagnóstico de la universidad refrendaba los obstáculos de una enseñanza impartida por facultades

"atrasadas, caducas en sus métodos esencialmente teóricos, con un profesorado casi sin remuneración e integrado en su mayor parte por los más connotados profesionales del país, muy eminentes sí, pero algunos

24 Guillermo Tello Rengifo, “La Democratización de la Enseñanza Universitaria”, Revista Jurídica 236 (1935): 1-2.

25 Álvaro Hernán Medina, Miguel Vásquez y Carlos Antonio Lis, "La reforma de la Universidad. Informe de la Comisión encargada de estudiar el proyecto de Ley Orgánica de la Universidad Nacional de Colombia”, Revista Jurídica 237-238 (1935): 281. 
de ellos sin siquiera medianas condiciones para catedráticos o expositores y todos con una explicable carencia de tiempo para dedicarle a su cátedra toda la consagración que ella requiere". ${ }^{26}$

Las condiciones y características del desempeño docente fueron considerados como uno de los principales obstáculos para constituir una Facultad que respondiera a las funciones universitarias, que generara conocimientos científicos pertinentes para la vida y la acción profesional. Esto incidía en una particular desvalorización de los títulos, dado que certificaban un conjunto de conocimientos teóricos pero que no correspondían a un "bagaje intelectual y práctico útil".

En la Facultad Nacional de Derecho y Ciencias Políticas, la reforma universitaria se convirtió en un proceso directamente ligado al desarrollo de las cátedras, de sus profesores y métodos, así como del vínculo entre los estudios realizados y la vida profesional futura. La reforma convirtió en un problema político la enseñanza profesional; el sentido de la formación de abogados, en cualquiera de sus materias, se debatió y transformó de cara a las necesidades de la nación, la cultura universitaria, el espíritu, la ciencia y la pertinencia de los conocimientos que serían puestos en práctica:

Sería muy útil un curso corto, en el primer año, de información general [...] Asípodría formarse el alumno un concepto general previo sobre su carrera y no iría tan desorientado y tan por las nubes [...] Sería conveniente también que se estatuyera en la ley la provisión de unos cuantos cargos inferiores, en juzgados y Tribunales, por estudiantes de tercero o cuarto año en adelante [...] Todo esto y los trabajos prácticos en las propias facultades harían los estudios de Derecho más y más positivos y aplicados, con lo cual saldrían ganando los estudiantes y el mismo público, víctima muchas veces de la inexperiencia de los noveles abogados. ${ }^{27}$

26 Medina, Vásquez y Lis, La reforma de la Universidad, 280.

27 Guillermo Tello Rengifo, "La reforma universitaria", Revista Jurídica nº 237 - 238 (1935): 268. 


\section{CONCLUSIÓN}

El análisis de la cátedra de economía política de la Facultad de Derecho y Ciencias Políticas de la Universidad Nacional, en el período de reforma universitaria avanzado bajo el gobierno de López Pumarejo, permite delinear las características de la formación de intelectuales en un medio universitario intervenido por la acción del Estado. Valida la percepción de la reforma universitaria como un problema de gobierno que, al salir de la arena discursiva y de legitimación de proyectos políticos, se insertó en la vida de las Facultades y en su fase de ejecución de los cambios asegurados por la legislación, presionó por cambios en la enseñanza profesional. Entonces es pertinente preguntarse: ¿cuáles son los elementos que una cátedra pone de relieve en un contexto de cambio político-institucional? ${ }^{28}$

En primer lugar, se debe considerar que la cátedra articula directamente -sin mediación- la relación entre profesores y estudiantes con el gobierno estatal, en un contexto en el que los órganos de gobierno universitario son débiles o se están constituyendo, como fue el caso de las propias Facultades y del Consejo Directivo y del Consejo Académico de la Universidad Nacional, de cuya labor dan testimonio los registros existentes desde el año 1936. La cátedra fue un espacio de expresión del contexto político del país y de la función que se le otorgó a la universidad en los proyectos desarrollo o modernización que impulsaron las fuerzas políticas y el Estado.

En segundo lugar, la cátedra permite identificar las tendencias de desarrollo de la disciplina que en ella se imparte, considerando los intereses docentes e intelectuales, los contenidos científicos y académicos, las redes y/o individuos participantes en la enseñanza. En este sentido, la cátedra de economía política en el período señalado se encuentra en el cambio de trayectoria de la reforma. La presencia del Presidente de la República como profesor es un indicador de la centralidad de los contenidos a impartir, y de la interpelación que por medio de ella se hace a la disciplina.

Los contenidos seleccionados en el programa y la estrategia de producción de un objeto epistemológico y de aprendizaje, indican la iniciativa de vincular la formación con los 'problemas nacionales' y, por ello, de la pertinencia de la

28 Bruno Bertherat, "L' élection à la chaire de médecine légale à Paris en 1879. Acteurs, réseaux et enjeux dans le monde universtaire", en Revue historique, n 644 (2007): 823-856. 
cátedra en la formación de abogados. Sin embargo, estos mismos contenidos fueron sometidos a crítica por su énfasis teórico y doctrinario en la enseñanza de la disciplina económica. La enseñanza de la economía, al insertarse de facto en la necesidad y legitimidad de la reforma universitaria, inició en el período su camino de singularización disciplinaria y su 'especialización profesional', hasta llegar en 1945 a la formación del Instituto de Ciencias Económicas aún adscrito a la Facultad de Derecho; y en 1952 a concretar su autonomía con la Fundación de la Facultad de Ciencias Económicas. Con ello, la reforma universitaria logró vincular orgánicamente la preocupación por los 'problemas colombianos', el interés nacional y la formación de economistas en la universidad pública. ${ }^{29}$

En tercer lugar, la cátedra es un criterio de evaluación de la trayectoria formativa de los estudiantes. El juicio sobre el carácter teórico de la formación y de los métodos implementados por los profesores -independientemente de las causas- se realizó desde los intereses por reforzar la "enseñanza profesional". Este aspecto evidencia el consenso existente sobre la necesidad de la reforma universitaria y, también, la articulación de la reforma con las condiciones y organización de la carrera del profesorado que se materializará en el Reglamento de 1937. Sin embargo, el problema central a considerar es que la "autonomía pedagógica" de la universidad hizo emerger los cuestionamientos sobre la idoneidad de los docentes para guiar a los estudiantes en una formación para la vida y la profesión, puso en duda sus capacidades para cumplir al mismo tiempo con la práctica de la ciencia y con su función social,con la innovación en métodos de enseñanza aportando a la "grandeza moral" de la Universidad Nacional.

\section{FUENTES}

Archivo Central Universidad Nacional de Colombia (AC UNAL), Bogotá, Colombia, Actas del Consejo Directivo 1936.

Archivo Central Universidad Nacional de Colombia (AC UNAL), Bogotá, Colombia, Nómina de Programas, proyectos, quejas, reglamentos 1927-1935.

29 Arturo Díaz, "La economía: su origen, sus motivos y la institucionalización de la enseñanza a nivel internacional, nacional y local. Siglos XVII - XX”, Revista Historia de la Educación Colombiana, nº11 (2008) 


\section{REFERENCIAS}

Arango, Eliseo. "Informe del Rector de la Facultad al Ministro de Educación Nacional". Revista Jurídica 236 (1935): 7-11.

Bayé. “La actual universidad. Progresos de 'Santa Clara'. El movimiento universitario. Entrevista con el doctor Rodrigo Jiménez Mejía”. Revista Jurídica 237-238 (1935): 278 - 279.

Bertherat, Bruno. "L' élection à la chaire de médecine légale à Paris en 1879. Acteurs, réseaux et enjeux dans le monde universtaire". Revue historique 644 (2007): 823-856.

Charle, Christophe. Naissance des “intellectuels". París: Les Éditions de Minuit, 1990.

Díaz, Arturo. “La economía: su origen, sus motivos y la institucionalización de la enseñanza a nivel internacional, nacional y local. Siglos XVII-XX". Revista Historia de la Educación Colombiana 11 (2008): 75-94.

El Tiempo. "López Pumarejo y su cátedra libre de Economía Política". En Alfonso López Pumarejo y la Universidad Nacional de Colombia, editado por la Universidad Nacional de Colombia, 37-44. Bogotá: Unibiblos, 2000.

García Prada, Carlos. "Exposición de motivos". En Alfonso López Pumarejo y la Universidad Nacional de Colombia, editado por la Universidad Nacional de Colombia, 69-91. Bogotá: Unibiblos, 2000.

Jaramillo Uribe, Jaime. "La educación durante los gobiernos liberales, 1930-1946". En Nueva Historia de Colombia, dirigida por Álvaro Tirado Mejía, 87-110. Bogotá: Planeta, 1989.

López Restrepo, Alejandro. Problemas colombianos. París: Editorial París-América, 1927.

Medina, Álvaro Hernán, Miguel Vásquez y Carlos Antonio Lis. “La reforma de la Universidad. Informe de la Comisión encargada de estudiar el proyecto de 'Ley Orgánica de la Universidad Nacional de Colombia'". Revista Jurídica 237-238 (1935): 279-282.

Montenegro, Álvaro. Los primeros programas de economía en Colombia. Bogotá: Universidad Javeriana, 2008. Consultado el 12-9-2012. http://www.javeriana.edu.co/fcea/ area_economia/inv/documents / LosprimerosprogramasdeeconomiaenColombia.pdf

Rhenals, Fernando. "Las ideas del Profesor Rollitz sobre la crisis económica mundial". Revista Jurídica 229-230 (1933): 134-139.

Restrepo, Félix. "Revista de libros: Recent social trends, Akerman, Becerra, Bruce, Edie, Einzig, Rollitz, Uribe". Revista Javeriana 2 (1933): 87-92.

Rollitz, Horst. "Problemas económicos colombianos". Revista Javeriana 3 (1933): 121127. 
Sánchez Botero, Clara. “Ciencia y educación superior en la República Liberal”. En República Liberal: sociedad y cultura, editado por Rubén Sierra Mejía. Bogotá: Universidad Nacional de Colombia, 2009, 519-555.

Tello Rengifo, Guillermo. “La Democratización de la Enseñanza Universitaria”. Revista Jurídica 236 (1935): 1-2.

Tello Rengifo, Guillermo. “La reforma universitaria”. Revista Jurídica 237-238 (1935): 265-268.

Molina Bravo, José. "Reforma, autonomía universitaria e interés nacional: análisis de la cátedra de Economía Política en la Universidad Nacional de Colombia, Bogotá 1933-1938". Revista Historia de la Educación Latinoamericana. Vol. 16 No, 22, (2014): 121-138. 